\title{
INTÉRÊT DE L'ÉTUDE DE LA FLORE DES DIATOMÉES DU LIEU DE SUBMERSION DANS LE DIAGNOSTIC DE NOYADE.
}

\author{
B. LUDES (1), M. COSTE (2), N. NORTH (1), P. MANGIN (1).
}

(1) Institut de Médecine Légale, 11 rue Humann, 67085 Strasbourg Cedex, France.

(2) CEMAGREF, 50 avenue de Verdun, B.P. 3, 33611 Gazinet Cedex, France.

\section{RÉSUMÉ}

La recherche de diatomées dans les tissus de la victime pour contribuer au diagnostic de noyade doit être accompagnée de l'analyse d'un échantillon d'eau prélevé sur les lieux de précipitation ou de découverte du corps. Cet échantillon permet d'établir un inventaire représentatif de la flore du lieu de découverte ou de submersion, et d'effectuer les comparaisons avec les taxons trouvés dans les tissus. De plus, la détermination de taxons dominants dans l'échantillon d'eau peut avoir une valeur indicative du lieu de découverte.

Mots-clés : submersion vitale, diatomées.

\section{INTEREST OF DIATOM FLORA INVESTIGATIONS AT DROWNING SITES IN THE DIAGNOSIS OF DEATH.}

\begin{abstract}
The diagnosis of drowning can only be made if the diatom investigation in the tissues of the victim is accompanied by the water sample analysis of the site of submersion or discovery of the corpse. The water samples allow to draw the flora profiling of the drowning site to be compared to the diatom genera found in the tissues. Moreover, the determination of the dominant diatom genera in the water sample can provide supportive evidence of the site of discovery.
\end{abstract}

Key-words : diagnosis of death, diatoms.

\section{INTRODUCTION}

La recherche et l'identification des diatomées dans les tissus d'une victime découverte dans l'eau est l'une des analyses biologiques nécessaire pour établir le diagnostic de noyade. En effet, quand la victime, lors de la submersion, effectue de grandes inspirations, les diatomées pénètrent avec l'eau dans les voies aériennes, dans les poumons. Elles passent ensuite, en raison de la rupture de la membrane alvéolo-capillaire, dans la circulation sanguine puis dans les organes. Ces algues peuvent donc être retrouvées dans les tissus de la victime, notamment dans la moelle osseuse en cas de noyade vraie, au contraire de l'immersion d'un corps où il ne peut plus y avoir de pénétration d'algues dans la circulation et dans les organes. 
Toutefois, cette méthode est controversée par différents auteurs, notamment SCHNEIDER (1980), GYLSETH et MOWE (1979), SCHELLMANN et SPERL (1979) et FOGED (1983). Ces auteurs ont observé, dans leurs séries, la présence de diatomées dans les tissus de sujets non noyés. Ils ont attribué cette présence d'algues soit à l'ingestion ou à l'inhalation de diatomées du vivant de la victime, soit à la pénétration passive de diatomées induite par la pression hydrostatique dans les cas d'immersion à plus de 3 mètres de profondeur.

D'après les cas de noyade étudiés depuis 1992 (60 cas) à l'IML de Strasbourg et en nous référant à AUER et MOTTONEN (1988), nous avons proposé des critères de positivité de l'analyse (LUDES et al., 1994). Une analyse peut être considérée comme positive si 20 diatomées sont retrouvées et identifiées dans $100 \mu \mathrm{l}$ de culot obtenu à partir de la digestion de $10 \mathrm{~g}$ de tissu pulmonaire et si 5 diatomées sont isolées à partir de $100 \mu \mathrm{l}$ de culot à partir de l'un des tissus suivants : tissus cérébral, rénal, hépatique et de la moelle osseuse.

Pour s'assurer de la provenance aquatique des diatomées, nous proposons une analyse qualitative d'un échantillon de diatomées provenant du cours d'eau où la victime a été découverte.

Dans ce travail, nous exposons les techniques de prélèvement in situ et les analyses des prélèvements de périphyton dans l'eau ainsi que leur comparaison avec des taxons extraits des tissus des victimes.

\section{MATÉRIEL ET MÉTHODES}

\section{Analyse des prélèvements de périphyton dans l'eau}

Les prélèvements sont réalisés par grattage d'une surface inerte de $20 \mathrm{~cm}^{2}$ pour recueillir les diatomées épilithiques. Les échantillons sont soumis à une incubation en présence d'eau oxygénée $(130 \mathrm{vol})$ à $80^{\circ} \mathrm{C}$ pendant 12 heures, pour éliminer toutes substances organiques. Après plusieurs lavages à l'eau MilliQ et une centrifugation finale à $2500 \mathrm{tpm}$ pendant $15 \mathrm{mn}, 100 \mu \mathrm{l}$ de culot sont étalés sur une lame porte-objet et fixés dans du Naphrax ${ }^{\circledR}$ pour observation au microscope optique à l'aide d'un objectif à immersion d'huile (Gross. 1000x). L'observation se fait sur 500 frustules afin de déterminer les taxons dominants dans l'échantillon d'eau. L'identification des espèces est réalisée selon la flore de KRAMMER et LANGE-BERTALOT (1986-1991).

\section{Analyse tissulaire}

Pour cette étude, nous avons analysé les tissus provenant de 30 corps repêchés dans les cours d'eau de la région strasbourgeoise.

Lors de l'autopsie des corps, $10 \mathrm{~g}$ de tissus pulmonaire, cérébral, rénal, hépatique et de moelle osseuse ont été prélevés. Les prélèvements sont réalisés en évitant toute contamination par l'eau des tables d'autopsie ou des instruments utilisés. L'extraction des diatomées fait appel à une digestion enzymatique par la protéinase $\mathrm{K}$, telle que nous l'avons déjà décrite (LUDES et al., 1994). $100 \mu$ l de culot sont étalés sur une lame porte-objet et fixés dans du Naphrax ${ }^{\circledR}$ pour l'observation microscopique.

\section{RÉSULTATS}

Cette étude a porté sur l'identification et le comptage des taxons dans les échantillons d'eau et dans les autres tissus de la victime. 
Le délai post morteri des corps découverts immergés est de 1 à 4 jours. Les diatomées ont pu être extraites de 22 échantillons de tissu pulmonaire, de 2 échantillons de tissu rénal, d'un échantillon de moelle osseuse et de tissu cérébral et de 3 échantillons de tissu hépatique.

Dans chaque cas où des diatomées sont présentes dans un tissu autre que le poumon, elles le sont également dans le poumon. Dans les tissus pulmonaires positifs (20 diatomées au moins), plus de 5 taxons différents ont été identifiés et de 49 à 64 diatomées $/ 100 \mu \mathrm{l}$ de culot $/ 10 \mathrm{~g}$ de tissu ont été dénombrées. Pour les autrès tissus, 3 taxons différents ont été retrouvés à l'analyse des lames considérées comme positives (5 diatomées au moins), avec une concentration maximale de 15 diatomées $/ 100 \mu \mathrm{l}$ de culot $/ 10 \mathrm{~g}$ de tissu.

Tableau I : Résultat de l'analyse des diatomées dans l'eau et dans les tissus des victimes.

Table I : Result of diatom flora found in the victims' water and tissues analysis.

\begin{tabular}{|c|c|c|c|c|c|c|}
\hline Cas & Eau & Poumon & Cerveau & Rein & Foie & $\begin{array}{c}\text { Moelle } \\
\text { osseuse }\end{array}$ \\
\hline 15 & + & + & - & - & - & - \\
\hline 2 & + & + & - & + & - & - \\
\hline 1 & + & + & - & - & - & + \\
\hline 1 & + & + & + & - & - & - \\
\hline 3 & + & + & - & - & + & - \\
\hline 8 & + & - & - & - & - & - \\
\hline
\end{tabular}

L'analyse quantitative montre que, dans 7 cas, les résultats sont positifs pour des tissus pulmonaires et non pulmonaires, et dans 15 cas ils ne le sont que pour le tissu pulmonaire (Tableau i). Dans 8 cas, aucune diatomée n'a pu être mise en évidence dans les échantillons tissulaires.

La figure 1 représente les taxons retrouvés dans un échantillon d'eau et dans un prélèvement pulmonaire. Les résultats sont exprimés en pourcentage par rapport au nombre total de taxons déterminés sur les lames (150 taxons pour le tissu pulmonaire et 500 taxons pour l'échantillon d'eau). Les taxons dominants dans l'eau ont également été retrouvés dominants dans le poumon : il s'agit de Navicula lanceolata pour les deux milieux, Stephanodiscus hantzschii et Navicula gregaria pour le poumon, alors que Stephanodiscus hantzschii, Navicula gregaria et Navicula radiosa sont présentes en concentration identique dans le prélèvement d'eau.

Une variation dans la flore des diatomées est observée en fonction du site et de la période de prélèvement. Les taxons retrouvés dans les tissus l'ont été, dans tous les cas, dans la flore du site où le corps a été retrouvé. Compte tenu de l'analyse quantitative, la dominance d'un taxon par rapport aux autres n'a pu être vérifiée que dans le tissu pulmonaire. 


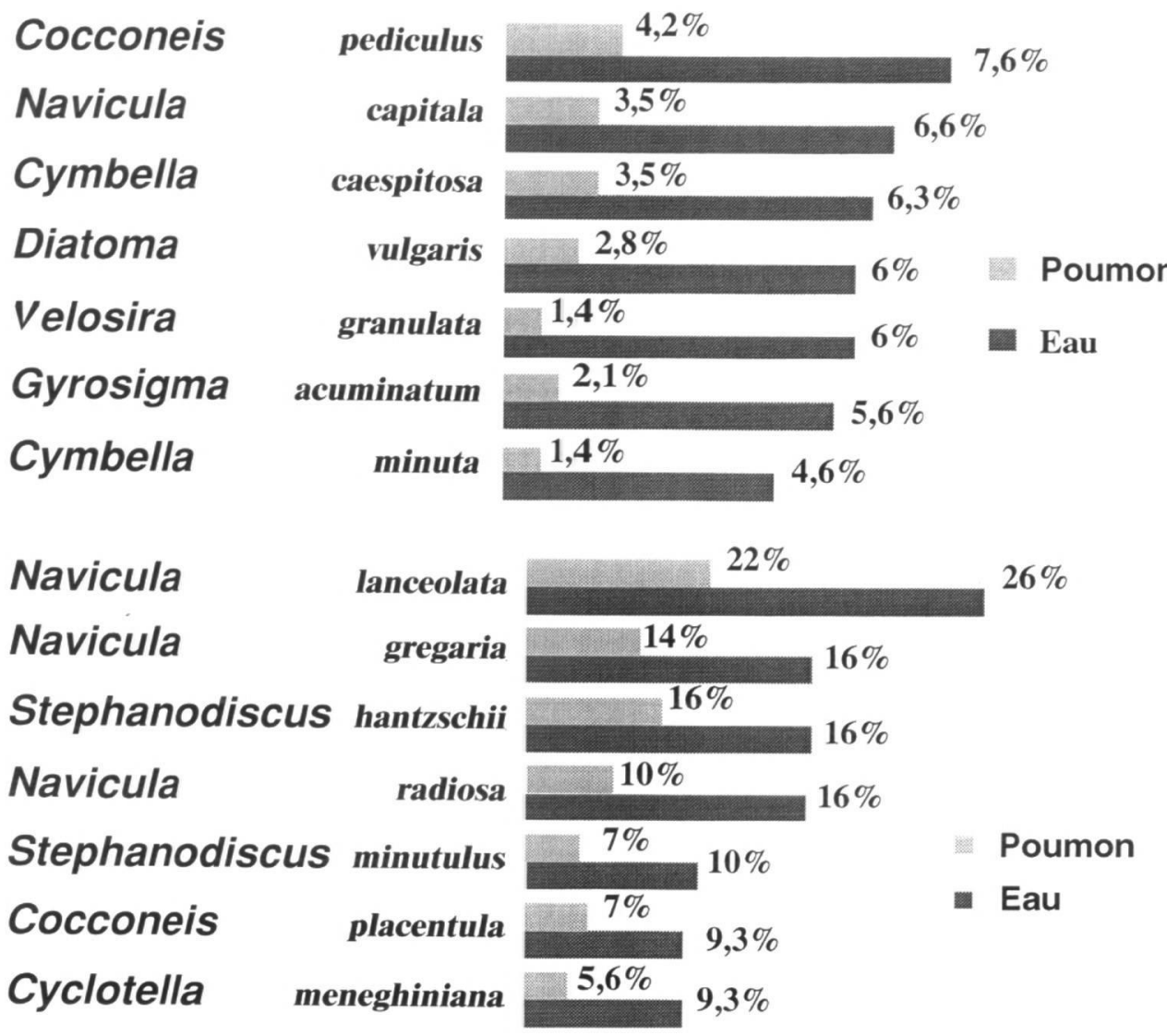

Figure 1 : Comparaison des taxons présents dans l'échantillon d'eau et dans le tissu pulmonaire d'une des victimes. Les résultats sont exprimés en pourcentages.

Les taxons sont représentés par ordre de fréquence décroissante dans l'échantillon d'eau.

Figure 1 : Comparison of the taxa found in one of the victims' water sample and lung tissue. The results are shown in percentages.

The taxa are shown in decreasing order of frequency in the water sample.

\section{DISCUSSION}

Les résultats de la recherche et de l'identification des diatomées doivent être interprétés en fonction de la possibilité de contamination par des diatomées provenant aussi bien des réactifs que de la pénétration passive sous l'effet de la pression hydrostatique durant l'immersion du corps dans l'eau. Cette pénétration passive n'atteint pas, pour les corps non putréfiés, les organes autres que le poumon et notamment la moelle osseuse. Pour permettre un diagnostic positif, il convient de rechercher ces algues dans les tissus de la victime (poumon, cerveau, rein, foie, moelle osseuse), mais également d'analyser un échantillon de diatomées provenant du lieu de découverte du corps ou du lieu supposé de la précipitation de la victime dans l'eau. Cette analyse permet de confirmer la provenance de diatomées retrouvées dans les tissus, d'éliminer des diatomées inhalées pendant la période ante mortem et de comparer les taxons dominants entre le tissu pulmonaire et l'eau d'immersion.

Dans cette étude, le diagnostic de submersion vitale a été retenu dans les 7 cas où l'analyse a été positive dans le tissu pulmonaire et dans les autres tissus analysés. Le diagnostic n'a pu être que présumé dans les 15 cas où seule l'extraction des algues des tissus pulmonaires a été positive (la pénétration passive n'étant pas à exclure). 
Toutes les diatomées retrouvées dans les tissus appartenaient à la flore aquatique prélevée à l'endroit et à l'époque de la découverte des corps.

\section{CONCLUSION}

La recherche et l'identification des diatomées dans les tissus de la victime permet de corroborer la submersion vitale dans les cas où les diatomées, en nombre suffisant, sont retrouvées aussi bien dans les tissus pulmonaires (20 diatomées/ $100 \mu \mathrm{l}$ de culot/ $10 \mathrm{~g}$ de tissu pulmonaire) que dans les autres tissus ( 5 diatomées/ $100 \mu \mathrm{l}$ de culot/ $10 \mathrm{~g}$ de tissu cérébral, hépatique, rénal et de moelle osseuse).

L'analyse des échantillons de diatomées épilithiques fournit des inventaires représentatifs de la flore des lieux de découverte ou de submersion de la victime. Ils permettent la comparaison des taxons découverts dans les tissus avec ceux trouvés dans l'eau. De plus, la détermination de taxons dominants peut aussi avoir une valeur indicative du lieu de découverte.

\section{REMERCIEMENTS}

Nous tenons à remercier Mademoiselle Anne TRICOT pour son aide dans la réalisation de ce manuscrit.

\section{BIBLIOGRAPHIE}

AUER A., MOTTONEN M., 1988. Diatoms and drowning. Z. Rechtsmed., 101, 87-98.

FOGED N., 1983. Diatoms and drowning. Once more. Forensic Sci. Int., 21, 153-159.

GYLSETH B., MOWE G., 1979. Diatoms in lung tissue. Lancet, 29, 1375.

KRAMMER K., LANGE-BERTALOT H., 1986-1991. Bacillariophyceae 1-4 in Süsswasser Flora von Mitteleuropas. 1, 876 p. ;2, 596 p. ; 3, 576 p. ; 4, 437 p. ; G. Fischer Verlag, Stuttgart.

LUDES B., QUANTIN S., COSTE M., MANGIN P., 1994. Application of a simple enzymatic digestion method for diatom detection in the diagnosis of drowning in putrefied corpses by diatom analysis. Int. J. Leg. Med., 107, 37-41.

SCHELLMANN B., SPERL W., 1979. Nachweis im knochen Mark (Femur) Nichtertrunkener. Z. Rechtsmed., 83, 319-324.

SCHNEIDER V., 1980. Detection of diatoms in the bone marrow of non-drowning victims. Z. Rechtsmed., 85 (4), 315-317. 